ज્પFRANÇAISE

$\supset \mathrm{DE}$

밈EAGOGIE

\section{Revue française de pédagogie}

Recherches en éducation

178 | janvier-mars 2012

Les politiques de lutte contre les inégalités scolaires d'un pays à l'autre

\title{
Les politiques québécoises d'intervention en milieux scolaires défavorisés : regard historique et bilan critique
}

Quebec's policy-making in disadvantaged school environments: A historical outlook and critical evaluation

Las políticas de intervención de Québec en entornos escolares desfavorecidos: mirada histórica y balance crítico

Interventionspolitik im benachteiligten Schulkontext in Quebec: historischer Überblick und kritische Bilanz

\section{Marc-André Deniger}

\section{(2) OpenEdition Journals}

Édition électronique

URL : http://journals.openedition.org/rfp/3555

DOI : 10.4000/rfp.3555

ISSN : 2105-2913

Éditeur

ENS Éditions

Édition imprimée

Date de publication : 15 mars 2012

Pagination : 67-84

ISBN : 978-2-84788-372-5

ISSN : 0556-7807

Distribution électronique Cairn

\section{CAIRN INFO}

CHERCHER, REPÉRER, AVANCER.

Référence électronique

Marc-André Deniger, «Les politiques québécoises d'intervention en milieux scolaires défavorisés : regard historique et bilan critique », Revue française de pédagogie [En ligne], 178 | janvier-mars 2012, mis en ligne le 15 mars 2015, consulté le 06 mai 2019. URL : http://journals.openedition.org/rfp/3555 ; DOI : 10.4000/rfp.3555 


\section{Les politiques québécoises $d^{\prime}$ intervention en milieux scolaires défavorisés : regard historique et bilan critique}

\section{Marc-André Deniger}

Ce texte propose une analyse historique et critique de l'évolution des politiques d'intervention québécoises en milieux défavorisés au cours des cinquante dernières années. II s'attarde d'abord à reconstituer l'évolution des principaux enjeux de ces politiques (conception du lien entre pauvreté et éducation, orientations et moyens, modes de gestion, répartition de rôles et pouvoirs, évaluation des résultats). Par la suite, il trace un bilan critique des politiques qui ont été menées afin de formuler, en s'inspirant des enseignements du passé, certaines suggestions susceptibles d'alimenter la réflexion sur l'avenir de ces politiques.

Mots-clés (TESE) : politique en matière d'éducation, milieu défavorisé, égalité des chances, décentralisation, évaluation du système éducatif, résultats de recherche.

\section{INTRODUCTION}

Le système scolaire québécois recherche depuis longtemps les orientations et les moyens qui lui permettraient de garantir l'« égalité des chances pour tous » et de minimiser l'influence de l'origine sociale sur la réussite scolaire. Déjà certaines recommandations du rapport Parent ${ }^{1}$ visaient à corriger des "inégalités de statut " attribuées aux régions et aux quartiers défavorisés (Ministère de l'Éducation du Québec, 1963, p. 84). Ce même rapport multipliait les recommandations relatives aux élèves en difficulté d'apprentissage ou qui connaissent des problèmes d'adaptation, promouvant par ce fait même l'établissement de mesures et de filières destinées à accueillir et à accompagner nombre de jeunes issus de milieux défavorisés. Depuis, différentes politiques adoptées tant aux plans national (ministère de l'Éducation du Québec, puis ministère de l'Éducation, du Loisir et du Sport du Québec) que régional (commissions scolaires, conseils scolaires) ont cherché à prendre le relais des intentions réparatrices déjà présentes dans le rapport Parent. Ces politiques ont, chacune à leur manière, suggéré une explication des principaux facteurs en cause dans l'échec scolaire, et cherché à fixer des objectifs et des moyens d'intervention susceptibles d'améliorer la situation.

Nous proposons ici une analyse historique de l'évolution des politiques d'intervention québécoises en milieux défavorisés au cours des cinquante dernières années ${ }^{2}$. Cette analyse critique puise ses sources dans une recension exhaustive de la docu- 
mentation relative aux politiques et aux périodes étudiées. Cette documentation se compose d'énoncés des politiques ou des programmes éducatifs, de mémoires, de plans d'action et de documents d'orientation (notre matériau premier), ainsi que des études et rapports (visant la description, l'analyse, l'évaluation, l'interprétation, etc.) qui y sont associés ${ }^{3}$. Afin d'approfondir la compréhension des enjeux à l'origine de ces politiques éducatives, nous avons interprété l'ensemble des sources documentaires en fonction des questions suivantes:

- quelles sont les populations visées par les politiques éducatives ? Qui les définit et comment les définit-on ? Comment pose-t-on le « problème » de la pauvreté et de ses conséquences quant à l'égalité des chances en éducation?

- quels sont les orientations générales, les objectifs et les mesures d'action promus et réalisés dans le cadre de ces politiques ? Observe-t-on une conformité entre la lettre et l'esprit des politiques et leur traduction en matière d'action concrète ?

- quel mode de gestion (centralisé ou décentralisé4) s'associe à l'application de ces mesures ?

- quels sont les rôles et les pouvoirs attribués aux différents acteurs touchés par l'application de ces mesures?

- quelle évaluation a été faite des politiques et des mesures d'action qui les fondent? Qui a conduit ces évaluations et quelles sont les principales recommandations qui s'en dégagent?

Notre itinéraire critique proposera, dans un premier temps, une analyse de ces enjeux et de leur évolution à travers un bref regard rétrospectif permettant de distinguer cinq périodes historiques (dont le découpage est fait en fonction des changements majeurs d'orientation des politiques gouvernementales) allant du début des années soixante à nos jours. Par la suite, nous tracerons un bilan critique des politiques qui ont été menées afin de formuler, en nous inspirant des enseignements du passé, certaines suggestions susceptibles d'alimenter la réflexion sur l'avenir de ces politiques.

\section{BREF REGARD RÉTROSPECTIF SUR L'ÉVOLUTION DES POLITIQUES}

\section{Un début : mesures compensatoires et adaptation des milieux défavorisés à l'école (1965-1974)}

Deux conceptions idéologiques de la relation entre l'école et les milieux défavorisés peuvent être déga- gées de cette première période. La première se fonde sur une « idéologie de la déficience ", les milieux défavorisés regroupant des individus " déficients " pouvant bénéficier de l'intervention de professionnels spécialisés ${ }^{5}$; la deuxième conception, dite de " guerre à la pauvreté ", invoque une explication plus collective et plus mobilisatrice, expliquant le fort niveau d'échec scolaire observé en milieu populaire par les inégalités liées à l'origine sociale. Bien qu'opposées, ces deux conceptions se côtoient dans les principaux documents de l'époque, dont la première politique de la commission des écoles catholiques de Montréal (CECM, 1970) en milieux défavorisés, l'« Opération renouveau ».

Cette politique se fonde toutefois davantage sur une philosophie compensatoire qui attribue des déficiences aux individus provenant de ces milieux. Elle établit également une gradation dans le niveau de "défavorisation " des écoles, certaines devant alors faire l'objet de mesures intensives. Cette politique cherche également à ajouter aux équipements disponibles, à appuyer le travail des enseignants et à assurer la coordination des ressources. Bien que plusieurs acteurs soient conviés à participer à l'Opération renouveau, tous ne se voient pas accorder une place équivalente. Le rôle principal est tenu par les administrateurs scolaires (conception et encadrement des programmes) et par les professionnels de l'éducation (interventions individuelles), alors qu'un rôle plutôt effacé, voire subalterne, est réservé au personnel enseignant. Les travailleurs sociaux deviennent, eux, des agents scolaires chargés de tâches spécialisées réalisées à l'intérieur de l'école. Les familles, présentées comme ayant un rôle dynamique, sont en réalité davantage l'objet d'interventions et peuvent difficilement agir sur leurs conditions et la réalité de l'école. Les universitaires sont appelés à valider les modèles d'organisation, à former le personnel ou à mener des études pouvant aider à mieux répondre aux besoins du milieu. Les termes clés de cette période politique étant la planification, la coordination, la rentabilité et l'efficacité de l'intervention, on accorde une importance considérable aux instances les mieux placées pour exercer ces opérations. Cette concentration de pouvoirs à l'extérieur des mains des acteurs plus proches de l'action est rapidement taxée de centralisatrice et de bureaucratique. Elle a d'ailleurs été battue en brèche, tant la volonté d'autonomie des écoles et de décentralisation des pouvoirs était grande : on observe un rééquilibrage en faveur des écoles avant même que le plan quinquennal n'ait été complété. C'est ainsi que, dès 1974, des " projets locaux » prenant appui sur l'action des directions d'école et du personnel ont 
vu le jour, s'imposant rapidement comme la « voie du succès » aux yeux des responsables politiques. Bien que la politique scolaire qui sous-tend l'Opération renouveau ne comporte pas de cadre formel d'évaluation, les observations faites à l'époque ont conduit à modifier les programmes, à revoir le modèle idéologique (de la « déficience » à la « différence ») et à modifier le rôle de l'institution scolaire (canalisatrice de l'action en partenariat). Trois recherches importantes doivent être signalées ici.

La première (Caouette \& Bourbeau, 1976) conteste les supposées carences des jeunes issus des milieux défavorisés : ils ne présentent pas de déficience cognitive particulière en termes de capacités linguistiques d'ordre expressif ou réceptif, de complexité du langage, de capacité d'attention, d'opérations de sériation et de classification, ou de compréhension des opérations mathématiques. Cependant on relève que les élèves de milieux défavorisés réagissent différemment aux stimulations sociales, ce qui les conduirait, notamment, à s'auto-évaluer de manière beaucoup plus sévère et à se croire promis à un moins grand succès que leurs pairs. La seconde étude, conduite par un groupe de travail de la CECM (1970), souligne également les limites de l'idéologie de la déficience et recommande un changement dans la philosophie et dans les stratégies d'intervention, recommandation qui sera appliquée par la suite. De l'avis des auteurs, si certaines mesures ont rencontré des difficultés, elles n'en méritent pas moins d'être reconduites et améliorées (mesures prévues auprès des familles et des jeunes enfants, prévention de l'effet d'étiquetage, etc.). Une troisième recherche (Bonnier-Tremblay, 1977) confirme l'absence de fondements de l'idéologie de la déficience et conclut que la majorité des enfants de milieux défavorisés se développent aussi normalement que ceux de milieux moyens ; on ne peut donc pas attribuer l'échec scolaire en milieux défavorisés à une plus grande incidence d'enfants présentant des retards de développement. Ce même rapport démontre que les mesures préscolaires compensatoires favorisées à l'époque ne parvenaient pas à influencer favorablement le développement des enfants. Cette conclusion, aussi mal accueillie par l'administration scolaire que par les enseignants, n'en confirmait pas moins les résultats des nombreuses recherches américaines, anglaises, françaises et québécoises qui mettaient alors en doute la pertinence des programmes d'éducation compensatoire, les effets de ceux-ci étant soit inexistants, soit peu durables (Bernstein, 1970 ; Isambert-Jamati, 1973).

\section{L'ancrage : respect des différences, adaptation de l'école à son milieu et projets locaux (1975-1987)}

La seconde période historique est caractérisée par une inversion de la philosophie et du système d'action observés durant la période précédente. Les facteurs sociaux à la base de la marginalisation de certaines populations scolaires sont maintenant reconnus. L'école se présente alors comme un partenaire actif au sein d'une lutte plus globale contre la pauvreté. Les orientations générales de ces politiques sont fondées sur le respect des différences, sur la discrimination positive en faveur des plus démunis et sur le pouvoir d'agir des communautés locales. C'est le milieu (ou la communauté) qui est conçu comme le principal médiateur des actions à entreprendre afin de favoriser l'apparition de conditions sociales promouvant l'égalité des chances en matière scolaire. L'école publique, pour sa part, doit s'adapter à son milieu et servir d'instrument de promotion - plutôt que de sélection - sociale. Cette adaptation se traduit autant en matière de "rapprochement " auprès d'élèves acceptés dans leurs différences que d'" ouverture " auprès des groupes et des organisations actifs en milieux défavorisés.

Les politiques mises sur pied au cours de cette période émanent de trois niveaux distincts : l'État québécois, les instances régionales (principalement le conseil scolaire de l'île de Montréal, CSIM par la suite) et les commissions scolaires ${ }^{6}$. L'État s'engage à accroître les fonds, à aider au développement de ressources didactiques et d'instruments d'évaluation et à financer des projets de perfectionnement de l'équipe-école. Cette allocation de ressources supplémentaires est encadrée par une nouvelle méthode de planification et de gestion budgétaire. Ainsi, sur le territoire montréalais, le conseil scolaire de l'île de Montréal est chargé d'identifier les milieux défavorisés, d'effectuer un classement des écoles en fonction de leur niveau de " défavorisation " et de partager les budgets selon les plans d'intervention. Les sommes sont allouées aux écoles les plus marquées par la pauvreté, en proportion du nombre d'élèves qu'elles accueillent?.

Les enseignants sont appelés à se familiariser avec la réalité des familles défavorisées. Le rôle de la direction de l'école doit gagner en importance. L'apparition de projets locaux amène la reconnaissance du rôle central joué par la direction quant à la traduction dans les faits des principes et des orientations des politiques. Les parents, quant à eux, sont appelés à s'impliquer davantage dans la vie de l'école et de leur commission scolaire, ce qui suppose un effort 
particulier de soutien, étant donné la distance considérable séparant les parents défavorisés de l'école. Cet effort a pris la forme d'interventions favorisant la complémentarité de l'action éducative des parents et de celle de l'école, de même que la participation des parents au fonctionnement de l'école et aux décisions prises en son sein. Cette logique de décentralisation exige toutefois que des contrôles et des supervisions continuent d'être menés par les autorités supérieures. Ces contrôles ont des conséquences quant au choix des interventions favorisées localement. Une décision locale suppose également une bureaucratisation. Ainsi, la marge de liberté et d'action concédée est soumise à de nombreux contrôles et paramètres. La commission des écoles catholiques de Montréal ${ }^{8}$ est soumise au contrôle du CSIM en matière de financement, de surveillance, de représentation, d'orientation, de coordination et de soutien aux projets existants. Le CSIM doit rendre des comptes au ministère de l'Éducation du Québec (MEQ par la suite) au sujet de l'utilisation des sommes octroyées et de l'application des politiques et des orientations ministérielles. II produit annuellement des bilans d'activité qui présentent des mesures de contrôle des objectifs généraux poursuivis par les programmes, en lien avec les moyens mis en œuvre et leurs conditions d'implantation. Une analyse ministérielle de ces bilans révèle que l'argent investi dans chaque intervention poursuit les objectifs prévus et atteint les résultats souhaités.

Cette lecture de la situation contraste avec le bilan sévère tracé par la recherche universitaire. Ainsi, Hohl (1983, 1985) souligne que l'adaptation de l'école aux besoins du milieu est devenue graduellement une adaptation de l'école aux besoins de l'équipe-école, et que les formalités bureaucratiques ont favorisé une homogénéisation des actions et un rapport lointain des enseignants et enseignantes au projet pédagogique de leur école. Une absence d'initiative sur le plan local, doublée d'une logique de " saupoudrage » de fonds qui n'accorde aucune valeur particulière aux projets pédagogiques les mieux articulés ou encore aux écoles réussissant le mieux le maillage avec les organismes de la communauté, empêcherait ainsi l'éclosion de solutions de rechange qui auraient pu modifier la donne de l'enseignement en milieux défavorisés. Pour leur part, Montmarquette, Houle, Crespo et alii (1985) en viennent à n'attribuer qu'une efficacité modeste aux mesures encouragées, reconnaissant plutôt le caractère déterminant de variables sur lesquelles l'école n'a aucune prise (comme le fonctionnement intellectuel de l'enfant ou la scolarisation de la mère). On estime que ces mesures n'ont pas été très efficaces pour améliorer les résultats dans les matières de base et que les interventions, représentant approximativement $60 \%$ du budget (pour les "pré-maternelles " et les enseignants ressources), n'ont pas produit les effets escomptés. Ces résultats conduiront les auteurs à proposer d'investir dans des programmes structurés et d'intervenir pendant les premières années du primaire. Cette recommandation fonde l'orientation générale du troisième plan d'action de la CECM. L'acceptation des résultats de cette évaluation par les autorités de la CECM va vite soulever une controverse : le fait de ne pas considérer les perceptions des intervenants du milieu, de ne pas porter une attention particulière aux conditions de réalisation de l'implantation des programmes et de ne pas tenir compte des effets indirects des mesures appliquées allait vite être dénoncé.

\section{Le rattrapage : pédagogies efficaces et mesures d'appoint en milieux défavorisés (1988-1994)}

Les missions de l'école en milieu défavorisé au cours des deux périodes historiques qui ont été évoquées correspondent peu aux finalités qui lui seront attribuées au milieu des années quatre-vingt. En effet, la politique ministérielle de 1980 fut largement critiquée. Son coût élevé allait également mal s'agencer aux politiques de rigueur budgétaire imposées par le gouvernement à l'ensemble du système public. Cette période se prêtait bien davantage au désengagement de l'État, au retour à l'« école de l'excellence » et à la domination de l'idéologie individualiste. Signe de cette désaffection, les intellectuels se sont désintéressés du champ des inégalités sociales en éducation (peu d'études, de publications ou de prises de position publiques). Qui plus est, les actions conduites en milieux défavorisés l'ont été sur une base locale et peu intégrée, ce qui a engendré la diversification des expériences et des projets scolaires sans que l'on sache précisément jusqu'où ceux-ci étaient réellement neufs, utiles ou encore efficaces. Ainsi, il n'existe pas, pour cette période, une politique spécifique ou un document phare. Les documents de l'époque s'inscrivent en continuité avec les politiques antérieures. On cherche surtout à mobiliser les ressources et les moyens d'intervention afin de réduire les écarts existant entre milieux favorisés et défavorisés. Dans l'esprit de l'époque, l'école en milieu défavorisé est une école comme les autres, mais à laquelle il convient d'allouer des ressources additionnelles. Cette aide est conçue comme un soutien pour atteindre des objectifs mesurables (résultats scolaires, taux d'échec, de diplomation et d'absentéisme) similaires à ceux des écoles d'autres milieux. Cette nouvelle vision « uniformisante " naît dans un contexte néolibéral qui met 
en question l'efficacité et la rentabilité des interventions correctrices menées par un État centralisateur.

Cependant, au début des années quatre-vingt-dix, on observe une intention de remobiliser les acteurs sociaux et d'intervenir à plusieurs niveaux à la fois. La vaste campagne de la centrale de l'enseignement du Québec sur la réussite éducative ${ }^{9}$ (voir notamment CEQ, 1991) a contraint l'État et la société civile à agir devant une situation perçue comme un grave problème social. Ce passage de l'action locale à l'action globale peut aussi être situé en continuité avec le rapport Un Québec fou de ses enfants ${ }^{10}$ (Bouchard, 1991) et avec d'autres projets ambitieux concernés par l'exclusion grandissante de larges pans de la jeunesse québécoise. Cette mobilisation met en évidence la nécessité d'une concertation à tous les échelons pour mener la lutte contre la pauvreté (MEQ, 1991). Cette intention de mobilisation se vérifiera cette fois-ci à l'aune de résultats mesurables et d'un nouveau financement dans le domaine de l'éducation. Les politiques de cette période s'inscrivent donc dans un esprit de rattrapage. II s'agit, à partir de mesures correctrices appliquées en bas âge, d'habiliter l'élève au succès scolaire.

Ainsi, sur le plan local, le quatrième plan d'action de la CECM énonce des objectifs qui visent pour l'essentiel à augmenter le pourcentage d'élèves qui maîtriseront les objectifs obligatoires d'apprentissage en mathématiques et en langue maternelle ; à réduire les écarts de résultats dans ces deux matières; à augmenter le niveau d'implication des parents ; à augmenter le pourcentage des élèves qui seront promus au secondaire en classes régulières ; à augmenter, chez les enseignants, le niveau d'acquisition des connaissances et des compétences rattachées à une pratique pédagogique adaptée aux élèves de milieu socioéconomiquement faible; et enfin à augmenter le nombre d'élèves qui poursuivront leurs études d'une manière continue au secondaire (CECM, 1989, p. 13-14).

Sur le plan national, le ministère se fixe un objectif ambitieux : " II faut que d'ici cinq ans $80 \%$ de ces jeunes obtiennent leur diplôme d'études secondaires. " (MEQ, 1992, p. 3) Les trois lignes de force, qui concernent au premier chef l'élève et ses relations avec l'institution scolaire, consistent à accompagner l'enfant dans sa démarche éducative et à le soutenir dans ses difficultés, ses réussites et ses projets d'avenir, de sorte que la relation entre l'école et l'enfant soit la plus personnelle, la plus humaine possible ; à varier la pédagogie de façon à tenir compte du rythme d'apprentissage, des besoins et des intérêts de chacun ; et à rendre attrayante la vie scolaire (MEQ,
1992, p. 5). Des mesures additionnelles seront appliquées aux écoles de milieux défavorisés : soutien aux parents, service d'animation télévisuel, soutien alimentaire, entrée en maternelle à plein temps dès cinq ans, maintien des maternelles existantes qui accueillent des élèves dès quatre ans, etc. (MEQ, 1992, p. 37). Fait à noter, la formulation de ces mesures révèle certains glissements de sens. Les milieux défavorisés y sont par exemple décrits comme des milieux à risque, ce qui n'est pas sans rappeler l'ère de l'idéologie de la compensation.

L'équipe-école demeure l'actrice principale des interventions entreprises. L'importance des directions d'école et l'influence toujours grandissante du corps enseignant chargé de conduire les principales actions correctrices se confirment. Le personnel enseignant est appelé à jouer un rôle central : analyse des résultats scolaires, établissement d'un diagnostic, recherche de moyens pour améliorer la situation, mise en place de stratégies et évaluation des actions entreprises (CECM, 1989). Soulignons cependant le caractère de plus en plus effacé de la contribution attendue des parents. Ceux-ci sont désormais définis comme des participants consultés ou encore comme des spectateurs ou des objets de l'intervention. Par ailleurs, on observe une inflation certaine des structures chargées de régir l'intervention. On s'assure qu'un soutien additionnel sera donné aux projets locaux par le biais d'une structure de concertation d'organisations (associations de cadres, de directeurs d'école, de professionnels, etc.) qui cherchera à optimiser le financement des mesures et des services inclus dans la formulation des projets locaux. Ainsi, une nouvelle structure de gestion est implantée à la CECM. Celle-ci comporte trois niveaux : le conseil central de coordination, les comités locaux et le service des études. Une fois établie cette concertation entre les membres de l'équipe-école, leurs alliés locaux et les différents niveaux de gestion, il reste encore à inscrire cet organigramme complexe dans la relation qui lie la CECM au CSIM, qui conserve son rôle d'allocation des sommes et de surveillance des projets. Le MEQ annonce également son intention de participer à l'animation et au soutien des projets éducatifs de l'équipe-école. Cette " configuration locale " s'ajoutera aux structures d'orientation et de contrôle déjà existantes et conduira à d'importantes luttes qui façonneront la politique ministérielle qui verra le jour en 1997.

Concernant les évaluations, deux phénomènes doivent être soulignés. D'abord, il apparaît clairement que les évaluations négatives qui ont précédé ont été mal reçues par les instances locales. L'écart 
entre les bilans locaux et les évaluations universitaires a engendré un repli vers des formules d'évaluation interne. Seul le CSIM a continué de favoriser une stratégie d'évaluation qui laisse une place aux chercheurs universitaires. Or, ces mêmes travaux ont présenté des résultats nuancés et parfois contradictoires eu égard à l'efficacité des interventions. À titre illustratif, trois études méritent d'être mentionnées. Celles-ci composent le premier rapport de recherche présenté au conseil québécois de la recherche sociale et au conseil scolaire de l'île de Montréal (Tremblay, Hébert \& Lapointe, 2000). La première étude examine le lien entre les programmes d'éducation préscolaire et la performance et l'adaptation de l'enfant à l'école. Les résultats, très mitigés, ne démontrent pas une amélioration de la performance, des résultats et de l'adaptation des élèves qui ont fréquenté la maternelle à plein temps. De même, aucune différence n'est relevée entre les élèves ayant fréquenté une pré-maternelle dès quatre ans et ceux qui ont fréquenté la maternelle à cinq ans seulement. Pourtant la deuxième étude, qui évalue la contribution de différentes variables à la réussite scolaire d'enfants de milieux défavorisés ayant suivi le programme de maternelle à plein temps, indique que l'âge d'entrée à la maternelle et le sexe semblent affecter la performance des élèves. Les écoles de petite taille, les enseignants qui ont un niveau d'attente élevé à l'égard des élèves et un sentiment d'efficacité professionnelle profond ont aussi une influence positive. Par contre, les variables reliées au niveau d'expérience de l'enseignant, à sa perception du climat scolaire, à son degré de satisfaction au travail (portant notamment sur les ressources disponibles et les politiques éducatives face aux problèmes de discipline) n'apparaissent pas avoir d'impact significatif sur les résultats des élèves. La troisième étude, qui cherche à identifier les pratiques éducatives favorisant la réussite des élèves, ne permet guère d'identifier des modes de fonctionnement particuliers aux écoles très performantes, plus ou moins performantes ou encore peu performantes. Ceci illustrerait le fait que les interventions éducatives sont peu différenciées d'un établissement à l'autre.

\section{Néolibéralisme versus réaffirmation des intentions égalitaristes : la genèse d'une réforme (1995-2002)}

La période suivante est marquée par la tension entre l'idéologie néolibérale et l'espoir de réaffirmer l'esprit égalitariste du système scolaire québécois. Bien qu'il ne soit pas devenu hégémonique, le discours néolibéral n'est pas sans conséquence sur l'éducation et les milieux défavorisés (possibilité élargie du choix de l'école, concurrence entre les établissements, accent mis sur la responsabilité individuelle, publication annuelle d'un palmarès des écoles). L'influence de ce courant d'idées se traduit principalement dans une modification progressive de la stratification du système scolaire. En réaction à la concurrence accrue de l'école privée (subventionnée par des fonds publics à hauteur de $60 \%$ ), le secteur public d'éducation réagit en «bonifiant » son offre scolaire par l'introduction de projets éducatifs à vocation particulière (sections sport-étude, arts, sciences, écoles internationales) ayant pour caractéristique commune de sélectionner les meilleurs élèves. Ceci entraîne un élitisme croissant de l'école publique, une homogénéisation des publics scolaires et engendre éventuellement une nouvelle hiérarchie du système scolaire, désormais composé d'un système privé en concurrence avec un système public «écrémé », puis des écoles publiques où seront reléguées les élèves en difficulté scolaire (habituellement pauvres ou issus de l'immigration et présentant une faible maîtrise du français).

Plusieurs acteurs de la société civile et de mouvements sociaux cherchaient à faire contrepoids aux préceptes néolibéraux de concurrence, d'individualisme et d'excellence. Le seuil visé par la politique ministérielle, $80 \%$ de diplomation au secondaire dans un horizon de cinq ans, n'était pas en voie d'être atteint. La réussite scolaire et la lutte contre l'inégalité des chances en éducation paraissaient commander un renouvellement des pratiques. Plus encore, il s'agissait de définir un « nouveau modèle démocratique pour l'éducation » (nom officiel de la commission présentée ci-dessous). Cette demande d'une réflexion globale sur l'éducation est principalement portée par les organisations syndicales de l'éducation (Berthelot, 1999) et par la directrice du journal Le Devoir, Lise Bissonnette (1992, 1993). C'est ainsi que fut créée la commission des états généraux de l'éducation, dont les travaux s'échelonneront sur une période de plus de dix-huit mois (1995-1996). Le rapport final de cette commission réaffirme l'idéal égalitariste (la finalité étant la démocratisation de la réussite) et propose de rénover notre système d'éducation en mettant en œuvre dix chantiers prioritaires, dont plusieurs concernent l'éducation en milieux défavorisés. Le plan d'action ministériel pour la réforme de l'éducation, partiellement inspiré du rapport de cette commission, met l'accent sur une réforme curriculaire similaire à celles qui se déploient simultanément dans certains pays européens (conception socioconstructiviste, introduction de la notion de compétence, transformation des méthodes d'évaluation des apprentissages, organisation par cycle d'enseignement, etc., 
voir MEQ, 1997a, 1997b). II prend aussi assise sur des priorités telles que l'intervention précoce (maternelle dès quatre ans en milieux défavorisés), la décentralisation vers les écoles et l'ouverture de celles-ci dans leur communauté (création de conseils d'établissement, nouveau mécanisme de gouvernance), le rôle accru des parents dans la vie de l'école et un soutien spécifique à l'école montréalaise où se pose avec une plus grande acuité les problématiques de la pauvreté et de l'intégration des communautés culturelles.

Plus particulièrement, le «Programme de soutien à l'école montréalaise » sera voué à un brillant avenir. De toutes les initiatives étudiées dans le présent bilan, c'est probablement celle qui sera la plus couronnée de succès dans son intention de soutenir les milieux défavorisés et de favoriser la transformation des pratiques pédagogiques et de l'administration scolaire. Ce programme propose huit stratégies, chacune accompagnée de mesures particulières:

- petite enfance, comme l'entrée en maternelle à cinq ans à plein temps ou à quatre ans à mi-temps en milieux défavorisés ;

- primaire et premier cycle du secondaire, comme le soutien à l'apprentissage, la récupération (temps réservé aux élèves qui ont besoin d'une attention supplémentaire pour pallier des difficultés d'apprentissage) ;

- élèves de familles immigrantes, comme l'élaboration de nouveaux modèles de soutien parental ;

- préparation à l'emploi, comme l'accroissement des services d'insertion, les stages enrichis en milieu de travail ;

- formation professionnelle, comme l'information des élèves, les activités d'exploration professionnelle dès la $1^{\text {re }}$ secondaire ;

- collaboration de l'école avec son milieu, comme l'affectation d'agents de liaison ;

- actions complémentaires en matière d'éducation, de santé et de services sociaux, comme une meilleure concertation des intervenants, une meilleure harmonisation des actions ;

- accès aux ressources culturelles, comme par exemple faciliter l'accès à des activités culturelles dont la qualité et l'offre de service sont contrôlées.

Malgré ces bonnes intentions, ce sont bien davantage les équilibres politiques entre les divers acteurs qui sont bousculés que la conception du problème de la réussite et de l'échec scolaire, qui demeure intact dans son acception compensatoire centrée sur les caractéristiques de l'enfant. Rarement l'école est considérée comme un facteur clé dans l'explication du faible taux de diplomation. Le fait qu'elle crée de la compétition entre les élèves, qu'elle privilégie certains contenus curriculaires, certaines formes de capitaux culturels et sociaux, ou qu'elle valorise certains types de comportement n'est jamais souligné. Rarement l'école est présentée comme exerçant, en milieux défavorisés, une fonction de sélection. La pauvreté est plutôt comprise comme une donnée sociodémographique. La politique ministérielle consiste donc à consacrer simplement plus de ressources là où les besoins paraissent être les plus criants. Les milieux défavorisés et les enfants issus de ces milieux sont définis comme porteurs de « risques».

Dans le plan d'action ministériel, les choses changent surtout au niveau de la répartition des responsabilités. Les mesures lancées précédemment avaient conduit à une superposition des prérogatives des acteurs. Celle-ci, ainsi que les conflits qu'elle a pu entraîner, commandait une reconfiguration du système et une clarification des zones de pouvoir dévolues aux différents acteurs en présence. L'État québécois s'est chargé de réduire la sphère d'influence des institutions intermédiaires en éducation, notamment par la diminution du nombre de commissions scolaires et par la modification de leurs fonctions. Cette simplification des structures s'est traduite par une plus grande implication de l'État et par une autonomie accrue des écoles. Aussi voit-on se profiler un enjeu d'importance : une politique de déconcentration des pouvoirs valorisant la responsabilisation locale (Trottier, 2000) a été promue par un pouvoir central animé par des exigences d'efficacité et d'efficience de plus en plus marquées. Ainsi l'adoption d'une nouvelle structure de démocratie scolaire, le conseil d'établissement, accorde des pouvoirs nouveaux à l'ensemble des membres de la communauté éducative, ce conseil étant ici compris comme un instrument mis sur pied afin de consolider l'autonomie de l'école et de renforcer ses liens avec la communauté.

Pour leur part, les enseignants sont appelés à connaître une modification importante de leur rôle, qui nécessite aussi une adaptation : nouveau curriculum, nouvelles méthodes, nouveau matériel pédagogique, nouveaux modes d'évaluation, nouveau classement des élèves, nouvelle grille de répartition du travail au sein de l'équipe enseignante, nouvelle structure de pouvoir (notamment avec les conseils d'établissement). On insiste particulièrement sur le fait que les enseignants doivent se doter de moyens qui leur permettront autant d'adapter leur enseignement aux profils des jeunes qu'ils instruisent que d'évaluer la portée de leur intervention pédagogique sur ces jeunes (innovation et évaluation continue). Ces changements 
profonds requièrent une accentuation du leadership pédagogique des directions d'école, ce qui suppose un accroissement important de leur tâche et engendre une nécessité de perfectionnement.

C'est par le biais du conseil d'établissement que les parents sont, quant à eux, invités à participer à la vie de leur école. Les parents élus représentent l'ensemble des parents de l'école et se prononcent par vote sur des décisions susceptibles d'influer sur la vie à l'école. Toutefois, les parents de milieux défavorisés, particulièrement sur l'île de Montréal, sont sousreprésentés au sein de cette instance (même au sein des écoles les plus défavorisées) et semblent davantage considérés comme "objets " de l'intervention (comme dans le cas du programme de soutien aux compétences parentales) plutôt que comme participants à part entière. On leur demande de suivre de manière régulière les activités scolaires de leurs enfants. On souligne que ce suivi pourra être dynamisé par la contribution de groupes communautaires spécialisés dans le domaine du soutien aux familles qui ne sont pourtant pas conviées à participer activement à la politique scolaire.

Au chapitre de l'évaluation des interventions, les recherches persistent à démontrer une quasistagnation des résultats escomptés par ces politiques. Ainsi Terrisse, Lefebvre et Larose (2000) observent un important décalage entre l'intention de départ des interventions éducatives précoces et la réalité des actions conduites. Le lien entre le degré de « défavorisation » et la performance scolaire n'a pas été amoindri par la présence d'une intervention correctrice. Une deuxième étude (Crespo, Carignan \& Kandarakis, 1998) analyse l'impact de l'activité de l'enseignant ressource (affectation de tâches à des enseignants se penchant en priorité sur la réponse aux difficultés d'apprentissage) en milieux défavorisés. Les résultats de l'étude paraissent très positifs : on réhabilite cette mesure populaire, malmenée lors d'études précédentes (Montmarquette \& Houle, 1989) ; on confirme le caractère raisonnable des dépenses encourues ; on révèle certains résultats syndicalement intéressants (" plus le travail de l'enseignant ressource tend vers le plein temps, plus le travail est associé à des rendements supérieurs en mathématiques $»)$. Ces résultats doivent cependant être accueillis sous certaines réserves, notamment à cause des problèmes d'ordre méthodologique soulevés par les auteurs eux-mêmes. Une troisième recherche (Janosz \& Deniger, 2001), qui légitimera un nouveau changement de cap lors de la période subséquente, démontre que les programmes de prévention du décrochage à l'ordre secondaire sont assez efficaces pour accroitre la motivation et les résultats scolaires. Cependant ils le sont beaucoup moins pour réintégrer les jeunes dans le secteur régulier (et lutter contre le maintien dans des filières marginales). En outre, les effets bénéfiques de ces programmes semblent être de courte durée.

\section{De la réforme à nos jours : gestion axée sur les résultats et les échecs concomitants}

Ces dernières conclusions concordent avec une réorientation de l'intervention gouvernementale qui s'amorce en 2002, avec le lancement de la "Stratégie d'intervention agir autrement " (SIAA). II s'agit d'une stratégie d'intervention destinée aux 200 écoles secondaires les plus défavorisées du Québec. Elle est caractérisée par l'ampleur des moyens investis (250 millions de dollars canadiens sur 5 ans), par l'approche décentralisée qu'elle préconise et par le rôle crucial qu'elle accorde à l'évaluation des effets de la politique et de la qualité de son implantation. Ces mêmes conclusions concordent également avec la confirmation d'un changement de cap significatif sur le plan de la régulation du système éducatif. L'obligation de résultats en éducation devient le leitmotiv qui détermine de plus en plus les pratiques éducatives et évaluatives. Cette nouvelle rationalité de l'action publique, inspirée de l'idéologie du New public management ${ }^{11}$, se traduit principalement par une insistance accrue sur la planification stratégique, la reddition de comptes et l'obligation de résultats prenant comme forme principale la détermination de cibles (standards) et la signature de conventions de gestion entre le MELS, les commissions scolaires et les écoles. L'introduction de la gestion axée sur les résultats a considérablement modifié la donne dans les relations politiques entre les acteurs du monde scolaire. S'il y a consensus sur le principe, les oppositions sont nombreuses à l'imposition par le MELS de façons de faire qui devraient relever des régulations intermédiaires (commissions scolaires) ou des écoles. Plusieurs y perçoivent un mouvement de recentralisation des pouvoirs vers le sommet stratégique du système éducatif (le ministère). D'autres dénoncent la conception réductrice de l'efficacité et de l'efficience véhiculée par cette logique et craignent ses effets pervers potentiels ${ }^{12}$ (sélection accrue des élèves, tricheries pour améliorer les scores, enseignement centré sur la préparation aux tests, etc.). D'autres encore disent craindre l'hypertrophie des procédures de planification stratégique au détriment des activités pédagogiques et en appellent plus ou moins explici- 
tement à la résistance contre cette logique associée à une bureaucratisation excessive.

L'époque contemporaine est aussi caractérisée par la conjonction de trois situations problématiques : les aléas d'une réforme éducative qui bat de l'aile suite à l'implantation plus que partielle de ses principaux préceptes au cours des dix dernières années (par exemple peu d'applications réelles du concept de compétence, très faible croissance tangible du travail collégial des enseignants regroupés en cycles) ; une nouvelle politique d'évaluation des apprentissages qui peine à convaincre à cause de sa complexité (trop de compétences à évaluer, absence initiale de cadre de référence pour ce faire) ; et l'échec d'une politique agressive et malhabile d'intégration des enfants handicapés et en difficultés d'adaptation et d'apprentissage en classes régulières (intégration rapide, sans discernement des modes de remédiation appropriés et sans ajout de ressources suffisantes). Ces problèmes justifieront : un contrôle ministériel accru s'apparentant à un processus de recentralisation, le déboulonnement discret et progressif des fondements de la réforme (fini les compétences, retour aux notes, abandon de l'organisation par cycle d'enseignement, etc.) et un renforcement de la logique des standards (plans stratégiques, plans de réussite, conventions de gestion et de partenariat, etc.).

Cette plus récente période se clôt sur un bilan fort mitigé de la "Stratégie d'intervention agir autrement » présentée plus haut. En effet, le rapport final de l'évaluation de cette ambitieuse stratégie (Groupe de recherche sur les environnements scolaires, 2010) pose un ensemble de constatations qui laissent perplexe tellement elles rappellent de façon lancinante les leçons du passé. D'abord on dénote un faible niveau de mise en œuvre des orientations et moyens de cette stratégie après six ans et une implantation très variable en fonction de la qualité du soutien aux écoles. Point positif, on observe une amélioration significative du " potentiel éducatif » des écoles (climat de l'école, soutien fourni aux élèves en difficulté, diminution de la violence et des comportements perturbateurs, meilleure collaboration entre écoles et familles), mais aucun changement des pratiques éducatives : on reste en périphérie de la classe. Les effets positifs ont surtout trait à la socialisation (relations entre pairs et envers les enseignants, diminution de la consommation de drogues), mais ne se sont pas étendus aux apprentissages scolaires et à la motivation. En outre, on souligne que certaines conditions organisationnelles ont semblé faciliter l'adoption de pratiques promues par cette stratégie (école de taille restreinte, présence d'un soutien proximal et continu). Toutefois, si le tiers des écoles ciblées « ont davantage avancé dans la réalisation de la démarche structurée de la SIAA » (Groupe de recherche sur les environnements scolaires, 2010), la grande part ont fonctionné à rebours en prenant des décisions qu'elles inscrivaient par la suite dans leur planification. Elles ont répondu aux pressions des milieux défavorisés par une répartition " équitable » des ressources (lire " une dispersion "), et elles ont cherché à maintenir les pratiques et les ressources déjà existantes et à soutenir des interventions organisationnelles ou parascolaires beaucoup plus que des interventions pédagogiques centrées sur les apprentissages et la motivation des élèves. Enfin les chercheurs identifient des facteurs explicatifs de l'écart entre ce qui était souhaité et ce qui fut réalisé qui se rapprochent des connaissances scientifiques éprouvées sur les problèmes d'implantation (un processus de planification peu mobilisant, une responsabilité trop exclusivement centrée sur les directions d'établissement, un développement professionnel qui tarde à soutenir le changement de pratiques en classe, un soutien inconstant, une faible valorisation des connaissances issues de la recherche, voir Fuhrman, Clune \& Elmore, 1988 ; Fullan, 2001 ; Odden, 1991). Nous reviendrons sur plusieurs de ces constats dans le bilan critique qui suit.

\section{BILAN CRITIQUE ET PERSPECTIVES}

Cette analyse critique de la documentation (voir notamment en annexe 2) nous a permis de dégager ce qui nous semble constituer les principaux enjeux historiques de la formulation et de la mise en œuvre des politiques étudiées, soit la conception du problème de la pauvreté et de l'égalité des chances en éducation, les objectifs et les moyens d'action des politiques, le mode de gestion de l'intervention, la répartition des rôles et des responsabilités entre les acteurs concernés, et l'évaluation des interventions entreprises. Nous tenterons maintenant de tracer un bilan critique de l'évolution historique de ces enjeux, une lecture transversale en quelque sorte, et de formuler des alternatives possibles inspirées de ces constatations.

\section{Une conception à revoir}

On dénote, depuis cinquante ans, une tension historique entre deux conceptions philosophiques et théoriques de la relation entre l'école et les milieux défavo- 
risés. La première, que nous qualifions d'« idéologie de la déficience ", se fonde sur une approche compensatoire des carences ou des déficits individuels et vise l'adaptation de l'enfant aux normes, aux attentes et au mode de fonctionnement de l'institution scolaire. Elle s'oppose à celle de la "guerre à la pauvreté " ou du courant de l'amélioration de l'école (school improvement), qui tiennent davantage compte des déterminants structurels de la pauvreté et préconisent plutôt l'adaptation de l'institution scolaire, de ses normes et de ses pratiques aux milieux défavorisés. Cette conception compensatoire est soutenue par une technoscience d'inspiration épidémiologique (Lesemann, 1994) dont la dernière déclinaison en vogue exacerbe cette centration sur les caractéristiques des élèves en expliquant les difficultés qu'ils rencontrent par leur «immaturité scolaire », traduction boiteuse de la notion anglo-saxonne de school readiness qui, dans les deux cas, nous rapproche de l'inquiétante tendance à l'individualisation décrite par les auteurs français (Demeuse, Frandji, Greger et al., 2011).

Dans une perspective historique, il est manifeste que l'idéologie de la déficience tient le haut du pavé. L'idéologie de la différence et de l'adaptation de l'école à son milieu fut moins largement préconisée, et surtout très faiblement opérationnalisée. Cette prédominance du paradigme de la déficience mériterait d'être remise en cause à plusieurs égards. D'abord, elle doit être jugée à l'aune de ses résultats, qui demeurent mitigés, et des effets de stigmatisation que comportent les interventions. On peut également penser que cette prédominance a longtemps contribué à affranchir l'institution scolaire de sa contribution à la sélection sociale et à la production ou à la reproduction des inégalités, et à déresponsabiliser cette même institution quant aux solutions à apporter. En contrepartie, si tant est qu'une alternative davantage centrée sur l'adaptation de l'école à son milieu soit possible et souhaitable, la traduction de ce second paradigme en des formes concrètes de pédagogie, de pratiques éducatives et d'organisation et de gouvernance scolaires demeure une perspective complexe et fort peu explorée.

\section{Une cohérence à assurer}

Cette opposition des conceptions du rapport entre l'école et la " défavorisation » porte sur les fondements de l'intervention (comment définir et diagnostiquer le problème), mais aussi sur les priorités et les cibles d'intervention à privilégier. Sur ce chapitre, certaines grandes tendances peuvent être dégagées. D'abord on dénote, non sans un certain étonnement, que certains moyens d'action perdurent à travers le temps (par exemple les libérations de temps pour les enseignants ou les formations de courte durée), malgré des changements majeurs sur le plan des orientations des politiques, comme si ces mêmes manières de faire pouvaient convenir à toutes les fins, indépendamment du fait que ces orientations soient davantage individuelles ou communautaires. On constate aussi une difficulté persistante à définir de façon opérationnelle, au-delà du discours d'intentions, les orientations politiques favorisant une ouverture de l'école à son milieu. De plus, la comparaison historique fait apparaître une oscillation continue de la philosophie des politiques d'intervention entre une approche spécialisée (par exemple centrée sur un problème spécifique des élèves) et une approche multimodale (qui tienne compte de l'élève, de sa famille, de l'école, de la communauté, des micro-systèmes, macro-systèmes, exo-systèmes, etc.). Notre rétrospective permet d'identifier certains problèmes de cohérence des politiques. Ainsi on dénote dans certains cas l'absence d'un cadre théorique éprouvé (comment le problème est-il posé et compris ? Cette conception s'appuie-t-elle sur des connaissances éprouvées ?) ou de schéma directeur des politiques (quelles sont les caractéristiques d'un bon projet, les résultats escomptés, les indicateurs et les critères retenus pour l'évaluation ?). Plus fréquemment, il existe un décalage entre les orientations et les moyens privilégiés pour agir, y compris l'attribution des ressources, entraînant des problèmes d'efficacité et d'efficience.

Sur le plan de la cohérence externe, la principale lacune des politiques d'intervention qui ont été analysées est l'absence de liens et de stratégies intégrant des mesures politiques dans d'autres domaines d'intervention de la politique publique, mesures susceptibles d'améliorer les conditions socio-économiques des familles à faible revenu et de leurs enfants (comme l'emploi, le revenu, la fiscalité, le logement ou la politique familiale). Ce manque de cohérence entre les domaines pertinents de l'action publique surinvestit l'école dans sa mission de lutte contre la pauvreté et de promotion de l'égalité des chances.

Plusieurs réflexions et actions s'imposent pour augmenter la cohérence et combler les lacunes des politiques et des programmes. À l'étape de l'élaboration, il conviendrait d'abord de clarifier les théories fondatrices, les objectifs, les priorités et les résultats attendus des politiques. Des choix s'imposent aussi sur le plan des orientations fondamentales des politiques (tout n'est pas conciliable). II faut également s'assurer du caractère opérationnel des objectifs mis 
en avant par cette politique, expliciter les résultats escomptés et prévoir les mécanismes et les indicateurs nécessaires à l'évaluation de l'atteinte de ces objectifs. Sur ce point, une réflexion est nécessaire sur la nature des résultats attendus et sur les priorités sous-jacentes : sont-ils strictement quantitatifs ou également qualitatifs ? Visent-ils exclusivement l'augmentation des résultats scolaires et des taux de diplomation ou revêtent-ils d'autres dimensions du métier d'élève, telles que l'insertion socioprofessionnelle ou l'adaptation psychosociale ? Au niveau pratique, la mise en œuvre réussie des politiques scolaires d'intervention en milieux défavorisés requiert une gestion du changement, un contrôle de l'implantation et une reddition de comptes qui évite la bureaucratisation excessive. II convient également d'être clair et précis quant aux moyens. Quelles sont les ressources nécessaires à la réalisation des objectifs et des résultats visés ? Les moyens sont-ils suffisants et appropriés? Correspondent-ils aux fins poursuivies ? On doit également se préoccuper des dédoublements et de la dispersion des ressources (par exemple, un trop grand nombre d'écoles mises en concurrence pour l'appropriation des ressources). II faut enfin réfléchir aux effets pervers potentiels des interventions et $y$ remédier le cas échéant.

\section{Un meilleur équilibre à trouver}

Il existe une seconde tension historique dans ce champ d'intervention politique : celle entre la conception et la gestion centralisée et décentralisée des interventions et des ressources. Bien qu'elles reconnaissent le rôle central du MELS dans la conception des politiques éducatives, les instances régionales et locales revendiquent leur juste part des responsabilités et des pouvoirs en matière de sélection des projets et de gestion des ressources. Elles font également valoir l'importance des expertises du milieu pour adapter les formules proposées par le cadre politique général aux besoins spécifiques de l'école et de la région. En contrepartie, l'engouement renouvelé pour une valorisation de l'intervention locale fait souvent fi des risques associés à une gestion déconcentrée de l'intervention (peu de preuves de son efficacité réelle, danger de dispersion des ressources, concurrence indue entre les établissements, multiplication des projets spéciaux qui peuvent faire double emploi, etc.). La quête d'un nouvel équilibre hybride entre le ministère et les milieux locaux implique une connaissance approfondie de l'éventail des projets mis en œuvre dans les écoles et une évaluation de leurs effets et de leurs impacts, afin de distinguer les « approches gagnantes » et d'éviter de tomber dans le piège de la décentralisation excessive ou de répéter l'erreur consistant à « faire toujours plus la même chose », ce qui fut largement le cas avec la SIAA.

Par ailleurs, la relation entre l'autorité centrale et les pouvoirs locaux implique un régime de concurrence politique qui oppose entre eux les acteurs du monde éducatif pour le contrôle du développement de pratiques éducatives et des ressources. Le choix d'une approche sélective dans l'attribution des ressources fait de la définition de la méthode de désignation des écoles défavorisées un enjeu de cette concurrence politique. On pourra ainsi observer l'existence de pressions pour accroître le nombre des écoles désignées (afin d'accéder à des ressources supplémentaires), ce qui risque d'engendrer la dilution des ressources ou des problèmes d'équité. Et il semble bien que cette concurrence entre les régions et les acteurs pour l'appropriation des ressources se soit exacerbée à la suite de la rationalisation des commissions scolaires, et avec la déconcentration des pouvoirs de ces dernières vers les écoles. À ce titre, l'un des défis majeurs de la gouvernance du système éducatif consiste à créer puis à conserver un équilibre entre, d'une part, une attribution centralisée des ressources en fonction de critères sociodémographiques qui garantiraient une régulation équitable du système éducatif et, d'autre part, la reconnaissance des meilleures initiatives locales, des pratiques les plus efficaces et efficientes.

II ressort également de l'analyse que cette tension politique entre la centralisation et la décentralisation des décisions et de la gestion a donné lieu à la multiplication et à la superposition des paliers d'expertise, de contrôle et d'intervention, contribuant ainsi à une bureaucratisation de ce champ d'intervention des politiques éducatives. Cette bureaucratisation a engendré une certaine confusion dans le partage des rôles et des pouvoirs entre les divers acteurs du système, qui se traduit par certaines incohérences de coordination et par l'absence de mécanismes clairs et efficaces de reddition de comptes. La bureaucratisation s'est accompagnée d'une multiplication des intervenants dans le domaine de la prise en charge des problèmes liés à la pauvreté, d'une professionnalisation du champ, et de la montée d'un pouvoir technoscientifique (Hohl, 1985 ; Lesemann, 1994). L'arène politique semble donc caractérisée par un certain néocorporatisme (Stoesz, 1983 ; Offe, 1985). Cette constatation d'ensemble ouvre sur de multiples voies de réflexion. Nous en retenons trois. D’abord, indépendamment de l'approche privilégiée (plus ou moins décentralisée), une « dé-bureaucratisation » de ce 
champ d'intervention et une simplification de l'organigramme s'imposent. En particulier, il convient de clarifier le partage des rôles, des pouvoirs et des responsabilités entre les acteurs du monde de l'éducation ainsi que les mécanismes de reddition de comptes. La prégnance de ce néo-corporatisme suggère également de soulever de nouveau une épineuse question de philosophie politique : comment faire en sorte que le bien commun (la lutte contre la pauvreté et contre les inégalités scolaires et la promotion de l'égalité des chances en éducation) motive les actions des acteurs et prime sur les intérêts particuliers?

Enfin, sur le plan du mode de gestion adopté pour la mise en œuvre des politiques, l'établissement d'un tel nouvel équilibre de la gouvernance scolaire requiert une certaine sagesse inspirée des connaissances acquises sur les facteurs contribuant au succès de l'implantation des politiques éducatives. Citons, à titre d'exemple, ces constatations inspirées des travaux d'Odden (1991) :

- les réformes ambitieuses (objectifs, durée, portée, ressources) réussissent généralement mieux que les efforts ciblés ;

- la gestion du processus de changement au niveau local est un élément clé de la réussite ;

- les approches ayant déjà fait leurs preuves et celles qui se fondent sur les connaissances scientifiques éprouvées donnent toujours de meilleurs résultats que des initiatives relevant du sens commun;

- les approches centralisées (top-down) fonctionnent bien si, et seulement si, certaines conditions sont réunies, en particulier l'adhésion des acteurs aux finalités de la politique, l'implication des enseignants dans l'élaboration des stratégies d'implantation au niveau local, le soutien suffisant et continu fourni aux écoles et aux enseignants, et le leadership des directions d'école et des commissions scolaires ;

- il convient d'adopter des modes de gestion et d'allocation des ressources visant à soutenir l'implantation locale et, plus spécifiquement, l'appariement adéquat de l'attribution des ressources avec les capacités organisationnelles et individuelles à développer (par exemple une culture de formation professionnelle ou l'adaptation des pratiques pédagogiques) ;

- l'engagement des acteurs dans le changement peut être assuré par leur implication active et continue dans la planification et le processus de mise en œuvre ;

- les connaissances et le savoir-faire (craftmanship) des enseignants sont des facteurs importants du succès et, en ce sens, la formation initiale et continue, de même que le soutien en classe, consti- tuent des conditions sine qua non de la réussite de l'implantation d'une politique éducative.

\section{Une évaluation à renforcer mais également à repenser}

En ce qui a trait à l'évaluation des diverses politiques et mesures mises en œuvre jusqu'à tout récemment, un bilan relativement négatif s'impose. On s'étonne en effet du manque d'efforts systématiques consentis à ce chapitre, et de l'absence d'une culture d'évaluation dans le domaine pourtant prioritaire des politiques éducatives. Les évaluations existantes - en particulier les évaluations externes - s'avèrent assez sévères et n'attribuent que peu d'impacts substantiels aux programmes analysés. Les résultats d'ensemble, très similaires à ceux que l'on retrouve dans les recherches conduites sur le continent nordaméricain, sont mitigés. Ce dur verdict externe semble avoir longtemps entraîné un repli défensif des milieux de pratique. Cette résistance des acteurs du monde scolaire s'est surtout traduite par la promotion d'évaluations internes. L'engouement pour l'auto-évaluation semble, dans plusieurs cas, avoir donné lieu à une confusion entre l'évaluation de la satisfaction des intervenants à l'égard des projets auxquels ils participent et l'évaluation des effets des politiques sur l'école, les élèves et leur famille. On peut parler ici d'un détournement de l'objet d'étude ou, plus spécifiquement, d'une redéfinition du référentiel d'évaluation à des fins stratégiques institutionnelles (comme exemples de comportement, on peut citer le fait de refuser de s'engager, de masquer ses échecs, de protéger ses ressources et de se prémunir contre les critiques).

L'absence, la faiblesse ou le manque d'objectivité des évaluations conduisent souvent à ce qu'on répète certaines erreurs ou reproduise des formules d'intervention dont l'efficacité n'est pas éprouvée ou, pire encore, dont l'inefficacité est démontrée. Cette " amnésie historique » exacerbe les problèmes récurrents de conception et d'implantation des politiques; on peut se demander si les nouvelles approches innovent et tiennent compte des enseignements des initiatives précédentes, ou si elles ont davantage tendance à reproduire les erreurs du passé. Ce doute est d'ailleurs corroboré par le rapport final de l'évaluation de la "Stratégie d'intervention agir autrement " (Groupe de recherche sur les environnements scolaires, 2010). Cette vacuité historique sur le plan de l'évaluation a malheureusement des implications pratiques significatives. La méconnaissance des approches et des stratégies pédagogiques efficaces en matière d'in- 
tervention auprès des élèves issus de milieux défavorisés, malgré l'importance accordée à cet aspect des solutions (Gauthier, Melouki, Simard et al., 2004 ; Bissonnette, Richard \& Gauthier, 2005), constitue un véritable mystère. On saisit mal pourquoi ces pratiques ne sont pas davantage connues ou, lorsqu'elles le sont, pourquoi ne sont-elles pas davantage adoptées par les écoles. La diffusion des connaissances scientifiques sur les pratiques " gagnantes » et le soutien aux écoles apparaissent donc comme des voies d'action à privilégier. En outre, alors qu'une vision institutionnelle ou experte du problème est favorisée, le peu de cas qui est fait de la parole des premiers concernés et du rapport à l'école des familles et des enfants pauvres laisse perplexe. Comment faire émerger une explication du problème de la pauvreté à l'école qui s'enracinerait dans la perspective des milieux défavorisés ? Cet élément devrait être l'une des assises de tout projet d'une école qui serait à la fois adaptée à son milieu et tenue à des standards de qualité.

Reste enfin l'épineux problème des résistances engendrées par l'évaluation dans les milieux scolaires. Les efforts plus systématiques d'évaluations externes (audits externes) des politiques, largement inspirés du courant du school effectiveness, qui furent récemment déployés (par exemple dans le cadre de l'évaluation de la SIAA), de même que la montée d'une gestion axée sur les résultats associant l'évaluation à l'atteinte de résultats (voire de standards) semblent en voie d'exacerber ces craintes plutôt que les diminuer. Dans un tel contexte de méfiance, il convient de s'interroger sur la marche à suivre afin que les acteurs du monde éducatif se dotent d'une culture commune de l'évaluation des politiques, des programmes et des pratiques qui atténuerait les craintes, engendrerait un climat de confiance et encouragerait l'engagement des acteurs. L'étude historique des politiques scolaires d'intervention auprès des milieux défavorisés québécois suggère à cet égard quelques préceptes :

- fonder l'évaluation sur les notions d'efficacité, d'efficience et d'équité à des fins de qualité plutôt que de performance, ce qui implique de remettre en question la notion de résultat telle qu'elle est actuellement définie ;

- axer en partie le travail sur la qualité de la formulation et de l'implantation des politiques et des programmes ;

- préconiser une participation active, représentative, mais aussi substantielle des acteurs du monde scolaire ;

- promouvoir les approches évaluatives soutenant l'auto-évaluation, le développement des compétences des milieux et l'amélioration des résultats des écoles (donc davantage inspirées du school improvement que du school effectiveness) ;

- évaluer dans une perspective d'accompagnement, de régulation de l'action et d'ajustement plutôt qu'évaluer pour effectuer la simple mesure des résultats ;

- lier étroitement l'évaluation au développement professionnel.

Marc-André Deniger marc-andre.deniger@umontreal.ca Université de Montréal, faculté des sciences de l'éducation

\section{NOTES}

1 La Commission royale d'enquête sur l'enseignement dans la province de Québec, mieux connue sous le nom de commission Parent (du nom de son président), a fait état de la situation de l'éducation au Québec dans les années soixante. Son rapport, le rapport Parent, est publié en 5 tomes en 1963 et 1964. II suggère diverses réformes du système d'éducation québécois en proposant notamment : la création du ministère de l'Éducation du Québec (MEQ, devenu depuis peu le MELS suite à l'intégration du ministère du Loisir et du Sport), la scolarisation obligatoire jusqu'à l'âge de 16 ans, la création des collèges d'enseignement général et professionnel (CEGEP) en remplacement des collèges de l'époque dirigés par des religieux, la formation poussée des enseignants, l'accès facilité aux universités en dehors de toute appartenance sociale. On pourra consulter les cinq tomes du rapport Parent en ligne : http://classiques.uqac.ca/contemporains/quebec commission parent/commission parent.html (consulté le 25 février 2012). Pour plus d'informations sur le système scolaire québécois et son historique, son fonctionnement et ses enjeux, voir l'ouvrage de Proulx (2009). On trouvera également une brève description du système scolaire québécois en annexe 1.

2 Pour des raison évidentes de concision, nous ne pouvons pas exposer ici l'intégralité de notre analyse historique, mais un résumé de l'essentiel. Pour en savoir plus, on pourra se référer à Roy \& Deniger (2003) et Deniger (2009).

3 On trouvera en annexe 2 la liste des documents officiels étudiés. Les autres documents (mémoires, études, etc.) se retrouvent dans la liste des références bibliographiques.

4 Cet enjeu de décentralisation ou de centralisation a trait ici à la répartition des pouvoirs, des rôles et des ressources entre le ministère de l'Éducation du Québec (sommet stratégique du système scolaire québécois), les commissions scolaires (régulation intermédiaire territoriale regroupant les écoles primaires et secondaires, soit les ordres d'enseignement chargés des 11 premières années de scolarisation, 6 au primaire, 5 au secondaire) et les établissements scolaires.

5 II s'agit des différences qui sont mises en avant en termes misérabilistes : un manque, un déficit de l'individu vivant dans une culture de pauvreté socialement inadaptée et non une différence posée en termes culturalistes, c'est-à-dire un écart entre une culture d'origine et la culture scolaire. Dans la région montréalaise, les différences culturelles sont historiquement caractérisés à la fois par la classe sociale mais aussi par l'origine ethnique, ce qui n'est pas le cas hors de la métropole. 
6 Les commissions scolaires sont des institutions présentes dans toutes les provinces du Canada. Au Québec, l'origine des commissions scolaires, telles que nous les connaissons aujourd'hui, remonte à 1845. La commission scolaire est une véritable institution politique locale possédant les attributs d'un gouvernement décentralisé qui est doté d'une vie politique ; elle a un pouvoir de taxation par l'impôt foncier ; elle a juridiction sur un territoire propre elle est responsable devant la population locale par l'élection de ses commissaires au suffrage universel.

7 Les données relatives à la « défavorisation » en milieu scolaire sont calculées à l'aide de deux variables (voir en ligne : http://www.mels. gouv.qc.ca/sections/publications/index.asp?page=fiche\&id=956, consulté le 25 février 2012) : l'indice du seuil de faible revenu (SFR) et l'indice de milieu socio-économique (IMSE). L'IMSE est constitue de la proportion des familles avec enfants dont la mère n'a pas de diplôme, certificat ou grade (ce qui représente les deux tiers du poids de l'indice) et de la proportion de ménages dont les parents étaient sans emploi durant la semaine de référence du recensement canadien (ce qui représente le tiers du poids de l'indice). Le SFR correspond à la proportion des familles avec enfants dont le revenu est proche ou inférieur au seuil de faible revenu, qui se définit comme le niveau de revenu auquel on estime que les familles consacrent $20 \%$ de plus que la moyenne générale à la nourriture, au logement et à l'habillement. II fournit une information qui sert à estimer la proportion des familles dont les revenus peuvent être considérés comme faibles, en tenant compte de la taille de la famille et du milieu de résidence (région rurale, petite région urbaine, grande agglomération, etc.). Encore une fois, il faut souligner la spécificité montréalaise où se concentre fortement la pauvreté. Ainsi cette année, 116 des 165 écoles de la commission scolaire de l'île de Montréal affichent un IMSE entre 8 et 10, indiquant la plus forte concentration d'élèves pauvres du Québec.

8 II faut savoir que le système scolaire public québécois a maintenu un statut confessionnel (catholique ou protestant) jusqu'en 1996, année à laquelle un amendement constitutionnel a mis fin à cette confessionnalisation pour conférer un statut linguistique aux commissions scolaires (francophones ou anglophones). Ainsi la commission des écoles catholiques de Montréal est devenue la commission scolaire de Montréal.

9 Cette centrale syndicale regroupait à l'époque la vaste majorité des enseignants québécois des ordres primaire et secondaire.

10 Très important rapport sur le bien-être de la jeunesse québécoise, qui eut de nombreux échos dans les diverses composantes de la société.

11 La gestion axée sur les résultats est une approche de gestion fondée sur des résultats mesurables répondant aux objectifs et aux cibles définis préalablement en fonction des services à fournir. Elle s'exerce dans un contexte de transparence, de responsabilisation et de flexibilité quant aux moyens utilisés pour atteindre les objectifs (Secrétariat du conseil du trésor, 2009). La montée en puissance de la gestion axée sur les résultats dépasse largement le cadre du système éducatif. En fait, il s'agit d'un changement de cap qui a inspiré la réforme de l'ensemble de la fonction publique québécoise. Cela démontre bien à quel point le champ de l'éducation, qui possède sa propre logique interne, est aussi traversé par des forces extérieures étatiques et sociétales.

12 On trouvera une synthèse complète de ces critiques dans le document de la centrale des syndicats du Québec (2002).

\section{BIBLIOGRAPHIE}

BABY A., GUILBERT N. \& SAVARD L. (1994). Pour une écologie de la réussite éducative : une analyse sociopédagogique des projets soumis par les commissions scolaires de l'île de Montréal dans le cadre de l'an 1 du Plan d'action sur la réussite éducative. Sainte-Foy : Université Laval, Centre de recherche et d'intervention sur la réussite scolaire.

BERNSTEIN B. (1970). «Education cannot compensate for society ». New Society, vol. 387, n० 15, p. 344-347.

BERTHELOT J. (1999). "La réforme de l'éducation de base: des origines connues, des résultats incertains ». Options CEQ, no 18, p. 9-25.

BISSONNETTE L. (1992). "Pauvreté et décrochage, le cercle ». Le Devoir, 29 juin, p. 12.

BISSONNETTE L. (1993). « La souque-à-l'école ». Le Devoir, 28 août, p. A8.

BISSONNETTE S., RICHARD M. \& GAUTHIER M. (2005). "Interventions pédagogiques efficaces et réussite scolaire des élèves provenant de milieux défavorisés ". Revue française de pédagogie, no 150, p. 87-141.

BONNIER-TREMBLAY F. (1977). DEDAPAM : projet de recherche, $3^{e}$ et dernier rapport, 1971 à 1976. Montréal : Service des études de la commission des écoles catholiques de Montréal.

BOUCHARD C. (1991). Un Québec fou de ses enfants. Rapport du groupe de travail pour les jeunes. Québec : Direction des communications du ministère de la Santé et des Services sociaux.

BRAIS Y. (1998). Le poids de la défavorisation sur la réussite scolaire des élèves de l'île de Montréal. Montréal : Conseil scolaire de l'île de Montréal.
CAOUETTE C. \& BOURBEAU G. (1976). Recherche sur la psychologie de l'enfant de milieu défavorisé. Montréal : Conseil scolaire de l'île de Montréal.

CENTRALE DE L'ENSEIGNEMENT DU QUÉBEC (1991). Réussir à l'école, réussir l'école. Rapport de recherche no D9643-1. Québec : Centrale de l'enseignement du Québec.

CENTRALE DES SYNDICATS DU QUÉBEC (2002). La réussite ne se décrète pas. Mémoire présenté à la Commission parlementaire sur le projet de loi 124 modifiant la loi sur l'instruction publique. Québec : Centrale des syndicats du Québec.

COMMISSION DES ÉCOLES CATHOLIQUES DE MONTRÉAL (1970). Politiques de la CECM en milieux défavorisés. Montréal : CECM.

COMMISSION DES ÉCOLES CATHOLIQUES DE MONTRÉAL (1977). Plan d'action 1977-1982 de l'Opération renouveau rendant opérantes les politiques de la CECM en milieux socio-économiquement faibles adoptées le 4 mars 1976. Montréal : CECM.

COMMISSION DES ÉCOLES CATHOLIQUES DE MONTRÉAL (1989). Opération renouveau : 1988-1991, $4^{e}$ plan d'action. Montréal : CECM.

COMMISSION DES ÉTATS GÉNÉRAUX SUR L'ÉDUCATION (1996a). Les états généraux sur l'éducation 19951996. Exposé de la situation. Québec : Gouvernement du Québec.

COMMISSION DES ÉTATS GÉNÉRAUX SUR L'ÉDUCATION (1996b). Rénover notre système d'éducation : dix chantiers prioritaires. Rapport final de la Commission des états généraux sur l'éducation. Québec : Gouvernement du Québec. 
CONSEIL SCOLAIRE DE L'ÎLE DE MONTRÉAL (1991). Les enfants de milieux défavorisés et ceux des communautés culturelles. Mémoire au ministre de l'Éducation sur la situation des commissions scolaires de l'île de Montréal. Montréal : CSIM.

CRESPO M., CARIGNAN N. \& KANDARAKIS H. (1998). Étude d'impact de la mesure intitulée : "Enseignantressource en milieu défavorisé ". Résumé de la recherche. Montréal : Conseil scolaire de l'île de Montréal.

DEMEUSE M., FRANDJI D., GREGER D. \& ROCHEX J.-Y. (2011). Les politiques d'éducation prioritaires en Europe. Tome 2: Quel devenir pour l'égalité scolaire? Lyon : ENS Éditions.

DENIGER M.-A. (2009). "Itinéraire critique des politiques scolaires d'intervention auprès des milieux défavorisés québécois : enjeux historiques et prospectives ". In K. Frohlich, M. De Koninck, P. Bernard \& A. Demers (dir.), Les inégalités sociales au Québec : comprendre et agir. Montréal : Presses de l'Université de Montréal.

DROLET M. (1990). L'enseignement en milieu socioéconomiquement faible. Montréal : Service des études de la CECM.

FUHRMAN S., CLUNE W. \& ELMORE R. (1988). «Research on éducation reform: Lessons on the implementation of policy ". Teachers College Record, vol. 90, n० 2, p. 237-258.

FULLAN M. (2001). The new meaning of educational change. New York: Teachers College Press.

GAUTHIER C., MELOUKI M., SIMARD D., BISSONNETTE S. \& RICHARD M. (2004). Interventions pédagogiques efficaces et réussite scolaire des enfants provenant des milieux défavorisés. Une revue de la littérature. Québec : Fonds québécois de recherche sur la société et la culture.

GROUPE DE RECHERCHE SUR LES ENVIRONNEMENTS SCOLAIRES (2010). Aller plus loin ensemble. Évaluation de la "Stratégie d'intervention agir autrement ». Synthèse du rapport final. Montréal : Groupe de recherche sur les environnements scolaires.

HOHL J. (1978). « Guerre à la pauvreté et réorganisation scolaire : l'enjeu des milieux défavorisés à Montréal ". International Review of Community Development, n० 39-40, p. 133-153.

HOHL J. (1983). Vers une problématique des projets locaux dans les écoles de milieux socio-économiquement faibles. Montréal : Conseil scolaire de l'île de Montréal.

HOHL J. (1985). "Les milieux socio-économiquement faibles analyseurs de l'école ». In M. Crespo \& C. Lessard (dir.), Éducation en milieu urbain. Montréal : Presses de I'Université de Montréal, p. 75103.

HOUDE-NADEAU M. \& COTNOIR B. (1992). Problèmes nutritionnels et performance scolaire en milieux défavorisés. Rapport de recherche. Montréal : Conseil scolaire de l'île de Montréal.

HOUDE-NADEAU M., NADON S. \& RACICOT S. (1995). Évaluation des mesures alimentaires en milieux défavorisés. Rapport de recherche. Montréal : Conseil scolaire de l'île de Montréal.

ISAMBERT-JAMATI V. (1973). « Les handicaps socioculturels et leurs remèdes pédagogiques".
L'orientation scolaire et professionnelle, no 4, p. 303318.

JANOSZ M. \& DENIGER M.-A. (2001). Évaluation de programmes de prévention du décrochage scolaire pour adolescents de milieux défavorisés 1998-2000. Rapport de recherche. Montréal : Centre de recherche et d'intervention sur la réussite scolaire et Institut pour le développement social des jeunes.

LEGAULT G. (1993). Services éducatifs donnés aux enfants de quatre ans de milieu économiquement faible: effet sur la diplomation. Québec : Gouvernement du Québec.

LESEMANN F. (1994). "La pauvreté : aspects sociaux ». In F. Dumont, S. Langlois \& Y. Martin (dir.), Traité des problèmes sociaux. Québec : Éd. de l'Institut québécois de recherche sur la culture.

McANDREW M. (1993a). Un allié pour persévérer! Rapport de recherche sur la prévention de l'abandon scolaire via le technicien en assistance sociale. Montréal : Conseil scolaire de l'île de Montréal.

McANDREW M. (1993b). Bienvenue à la retenue scolaire! Rapport de recherche sur les études dirigées au primaire. Montréal : Conseil scolaire de l'île de Montréal.

MINISTÈRE DE L'ÉDUCATION DU QUÉBEC (1963). Rapport de la commission royale d'enquête sur l'enseignement dans la province de Québec, tome 1. Québec : Gouvernement du Québec.

MINISTÈRE DE L'ÉDUCATION DU QUÉBEC (1981a). Rapport final : évaluation de l'impact des interventions en milieu économiquement faible au niveau préscolaire. Québec : Gouvernement du Québec, direction générale du développement pédagogique.

MINISTÈRE DE L'ÉDUCATION DU QUÉBEC (1981b). Rapport-synthèse final sur l'évaluation des interventions éducatives en milieu économiquement faible. Québec : Gouvernement du Québec, direction générale du développement pédagogique.

MINISTÈRE DE L'ÉDUCATION DU QUÉBEC (1991). Notre force d'avenir : l'éducation. Orientations 1991-1993. Québec : Gouvernement du Québec.

MINISTÈRE DE L'ÉDUCATION DU QUÉBEC (1992). Chacun ses devoirs : Plan d'action sur la réussite éducative. Québec: Gouvernement du Québec.

MINISTÈRE DE L'ÉDUCATION DU QUÉBEC (1997a). Prendre le virage du succès: Plan d'action ministériel pour la réforme de l'éducation. Québec : Gouvernement du Québec.

MINISTÈRE DE L'ÉDUCATION DU QUÉBEC (1997b). Prendre le virage du succès : Soutenir l'école montréalaise, 1997-1998. Québec : Gouvernement du Québec.

MINISTÈRE DE L'ÉDUCATION DU QUÉBEC (2002). Agir autrement pour la réussite des élèves du secondaire en milieu défavorisé. Stratégie d'intervention pour les écoles secondaires. Québec : Gouvernement du Québec.

MONTMARQUETTE C. \& HOULE R. (1989). Les interventions scolaires en milieu défavorisé. Estimation et évaluation. Montréal : Presses de l'Université de Montréal. 
MONTMARQUETTE C., HOULE R., CRESPO M. \& MAHSEREDJIAN S. (1985). " L'impact des interventions éducatives en milieux économiquement faibles: le programme de l'Opération renouveau ". In M. Crespo \& C. Lessard (dir.), Éducation en milieu urbain. Montréal : Presses de l'Université de Montréal, p. 31-53.

ODDEN A. (1991). "New patterns of education policy implementation and challenges for the 1990s ». In Education policy implementation. New-York : State University of New York Press, p. 297-327.

OFFE C. (1985). « The attribution of status to public interest groups ". In Disorganised capitalism: Contemporary transformations of work and politics. Cambridge : MIT Press.

PROULX J.-P. (2009). Le système éducatif du Québec. De la maternelle à l'université. Montréal : Chenelière Éducation.

ROY G. \& DENIGER M.-A. (2001). Enquête auprès des membres votants des conseils d'établissement. Québec : Ministère de l'Éducation du Québec.

ROY G. \& DENIGER M.-A. (2003). Des mesures compensatoires à l'obligation de résultats : bilan critique des politiques scolaires d'intervention auprès des milieux défavorisés québécois. Note de recherche n० 49. Québec : Centrale des syndicats du Québec.

SAINT-JACQUES M. (1991). "L'égalité des chances et l'inégalité des ressources en milieux défavorisés : un tandem indissociable ». Apprentissage et socialisation, vol. $14, n^{\circ} 1$, p. 63-67.
SECRÉTARIAT DU CONSEIL DU TRÉSOR (2009). Modernisation de la gestion publique : guide sur la gestion axée sur les résultats. Québec : Secrétariat du conseil du trésor.

STOESZ D. (1983). "Corporate welfare : la réforme du welfare state aux États-Unis ". Revue internationale d'action communautaire, vol. 50, no 10 , p. 87-100.

TERRISSE B., LEFEBVRE M.-L. \& LAROSE F. (2000). Analyse des caractéristiques des mesures d'intervention éducative précoce et comparaison de leurs effets à moyen terme sur l'adaptation scolaire et sociale d'enfants de milieu socio-économiquement faible. Rapport final de recherche présenté au Conseil québécois de la recherche sociale pour la subvention, no 2677 095. Québec : Conseil québécois de la recherche sociale.

TREMBLAY R., HÉBERT M. \& LAPOINTE P. (2000). Impact des mesures d'éducation préscolaire en milieux défavorisés sur l'île de Montréal. Rapport de recherche présenté au CQRS et au CSIM dans le cadre du Programme d'action concertée sur la prévention du décrochage scolaire en milieux défavorisés. Montréal: Université de Montréal, Groupe de recherche sur l'inadaptation psychosociale chez l'enfant.

TROTTIER C. (2000). «Enjeux du nouveau partage des pouvoirs en éducation au Québec suite à l'implantation des conseils d'établissement aux niveaux primaire et secondaire ". Communication présentée au congrès de I'ACFAS, Montréal.

\section{ANNEXE 1. LE SYSTÈME D'ÉDUCATION DU QUÉBEC : UNE BRÈVE DESCRIPTION}

Cette description est extraite du site Internet du ministère de l'Éducation, du Loisir et du Sport (http://www. mels.gouv.qc.ca/scolaire/educqc/index.asp, consulté le 25 février 2012).

Au Québec, l'enseignement primaire dure normalement six ans et l'enseignement secondaire cinq ans. L'âge d'admission à la première classe de l'enseignement primaire est fixé à six ans révolus avant le 1er octobre de l'année scolaire en cours. La maternelle, pour les enfants de cinq ans, généralement fréquentée à mi-temps dans le passé, est à temps plein depuis l'automne 1997. Elle n'est pas obligatoire, mais presque tous les enfants y sont inscrits. L'enseignement public est donné par les commissions scolaires. Celles-ci sont dirigées par des commissaires élus par l'ensemble de la population qu'elles desservent. Les commissions scolaires engagent elles-mêmes le personnel dont elles ont besoin pour offrir des services éducatifs. L'enseignement primaire et l'enseignement secondaire sont également donnés par des établissements privés, certains étant subventionnés par le ministère de l'Éducation, du Loisir et du Sport. Les établissements d'enseignement privés rassemblent $7 \%$ des élèves du primaire et environ $19 \%$ des élèves du secteur des jeunes du secondaire.

Les études secondaires durent normalement cinq ans (secondaire 1 à secondaire 5). L'enseignement collégial offre des programmes conduisant au diplôme d'études collégiales (DEC) et des programmes courts en formation technique conduisant à une attestation d'études collégiales (AEC). La durée des programmes conduisant au DEC en formation pré-universitaire est, en principe, de deux ans et celle des programmes conduisant au AEC de trois ans.

L'enseignement universitaire se divise en trois cycles d'études. Le premier conduit notamment à l'obtention du baccalauréat, généralement après trois ans d'études, ou, moins souvent, après quatre ans dans certains programmes. Le deuxième cycle mène à l'obtention de la maîtrise et le troisième cycle à l'obtention du doctorat. Les universités décernent également des certificats, des diplômes et d'autres formes d'attestations pour sanctionner la réussite de programmes courts. En 2007-2008, le gouvernement du Québec a contribué à $53 \%$ du financement des universités. 


\section{ANNEXE 2. DOCUMENTATION OFFICIELLE RELATIVE AUX POLITIQUES ÉDUCATIVES SELON LES PÉRIODES ÉTUDIÉES}

\section{Mesures compensatoires et adaptation des milieux défavorisés à l'école (1965-1974)}

Conseil des œuvres de Montréal, Opération renouveau social. Stratégie en vue de réduire les inégalités socioéconomiques dans les zones défavorisées de Montréal, 1966.

CECM, Opération renouveau, 1970.

\section{Respect des différences, adaptation de l'école à son milieu et projets locaux (1975-1987)}

Commission des écoles catholiques de Montréal (CECM), Deuxième plan d'action de l'Opération renouveau (1977-1982).

CECM, Troisième plan d'action de l'Opération renouveau (1984-1987).

Conseil scolaire de l'île de Montréal (CSIM), Vers une politique du conseil scolaire de l'île de Montréal concernant les interventions scolaires en milieu socio-économiquement faible, 1977.

Ministère de l'Éducation du Québec (MEQ), L'école québécoise, énoncé de politique et plan d'action, 1979. MEQ, L'école s'adapte à son milieu : énoncé de politique sur l'école en milieu économiquement faible, 1980. MEQ, Une école communautaire et responsable, 1982.

\section{Pédagogies efficaces et mesures d'appoint en milieux défavorisés (1988-1994)}

CECM, Quatrième plan d'action de l'Opération renouveau (1988-1992).

CECM, Cinquième plan d'action de l'Opération renouveau (1993-1998).

CSIM, Mémoire sur la situation des enfants de milieux défavorisés et ceux des communautés culturelles, 1991. MEQ, Chacun ses devoirs : plan d’action sur la réussite éducative (plan Pagé), 1992.

\section{Réforme de l'éducation et changements récents (1995-2002)}

MEQ, Rapport de synthèse de la commission des états généraux sur l'éducation, 1996.

MEQ, Prendre le virage du succès (intervenir dès la petite enfance), 1997.

MEQ, Plans d'action du programme de soutien à l'école montréalaise, 1997, 1998, 1999, 2000.

MEQ, Plan d'action du programme de l'école orientante, 2000.

MEQ et Commission scolaire de Montréal, Stratégie d'intervention agir autrement, Opération solidarité, 2002. 
